\title{
1 Science Forum: Sex differences and sex bias in human circadian 2 and sleep physiology research
}

3 Manuel Spitschan 1, 2, 3, II, [0000-0002-8572-9268], Nayantara Santhi, II, [0000-0003-4568-1447], Amrita 4 Ahluwalia $^{5,[0000-0001-7626-6399]}$, Dorothee Fischer ${ }^{6,[0000-0002-2122-3938]}$, Lilian Hunt, 8, [0000-0002-0319-

$5{ }^{7764]}$, Natasha A. Karp ${ }^{9}$ [0000-0002-8404-2907] , Francis Lévi ${ }^{10,11,12 \text {, [0000-0003-1364-7463] }}$, Inés Pineda6 Torra $^{13,[0000-0002-7349-2208]}$, Parisa Vidafar ${ }^{14,15}[0000-0002-3990-1047]$, Rhiannon White ${ }^{3,10,[0000-0002-}$ 7 8175-3586],

8

$9{ }^{1}$ TUM Department of Sport and Health Sciences (TUM SG), Technical University of 10 Munich, Germany

112 Translational Sensory and Circadian Neuroscience, Max Planck Institute for Biological 12 Cybernetics, Tübingen, Germany

$13{ }^{3}$ Department of Experimental Psychology, University of Oxford, United Kingdom

$14{ }^{4}$ Department of Psychology, Northumbria University, United Kingdom

$15{ }^{5}$ William Harvey Research Institute, Barts \& The London School of Medicine \& Dentistry, 16 Queen Mary University of London, United Kingdom

$17{ }^{6}$ German Aerospace Center, Institute of Aerospace Medicine, Sleep and Human Factors 18 Research, Germany

$19 \quad{ }^{7}$ Wellcome Trust, United Kingdom

$20 \quad{ }^{8}$ Equality, Diversity and Inclusion in Science and Health Group, United Kingdom

Note: Authors after the first two authors are listed in alphabetical order.

${ }^{9}$ Data Sciences \& Quantitative Biology, Discovery Science, R\&D, AstraZeneca, United Kingdom

${ }^{10}$ Warwick Medical School, University of Warwick, United Kingdom

${ }^{11}$ Hepatobiliary Center, Hospital Paul Brousse (AP-HP), France

12 UPR “Chronotherapy, Cancer and Transplantation”, Medical School, Paris-Saclay University, France

${ }^{13}$ Centre for Cardiometabolic and Vascular Science, Division of Medicine, University College London, United Kingdom

${ }^{14}$ Sleep and Circadian Research Laboratory, Department of Psychiatry, University of Michigan, United States

15 School of Psychological Sciences and Turner Institute for Brain and Mental Health, Monash University, Australia

To whom correspondence should be addressed: $\mathrm{Dr}$ Manuel Spitschan (manuel.spitschan@tum.de, manuel.spitschan@tuebingen.mpg.de) and Dr Nayantara Santhi (nayantara.santhi@northumbria.ac.uk) 


\section{Abstract}

41 Growing evidence shows that sex differences impact many facets of human biology. Here we 42 review and discuss the impact of sex on human circadian and sleep physiology, and we 43 uncover a data gap in the field investigating the non-visual effects of light in humans. A 44 virtual workshop on the biomedical implications of sex differences in sleep and circadian 45 physiology then led to the following imperatives for future research: (1) design research to be 46 inclusive and accessible, (2) implement recruitment strategies that lead to a sex-balanced 47 sample, (3) use data visualization to grasp the effect of sex, (4) implement statistical analyses 48 that include sex as a factor and/or perform group analyses by sex, where possible, (5) make 49 participant-level data open and available to facilitate future meta-analytic efforts. 
51 Despite marked sex differences in many aspects of human physiology and behaviour, 52 biomedical research continues to be disproportionately biased towards the male sex. For example, women made up only $25 \%$ of participants in landmark trials for congestive heart failure and $19.2 \%$ of participants for studies in antiretroviral treatment of HIV [1]. Such a skewed evidence base leads to disparities in clinical and non-clinal research applications, and weakens the impact of science-based policies and translational outcomes.

This sex bias or 'sex data gap' - whereby data mainly come from male individuals - has recently received widespread attention [1], with policy advisers [2], funders [3, 4] and publishers $[5,6]$ pushing for better inclusivity in research regarding sex. Embracing these new practices should improve translational outcomes and scientific efficiency, but this will require a two-pronged tactic that both strengthens forces for change and weakens barriers in the field [7]. The problems that allow sex bias to emerge are multifaceted and closing the data gap will require solutions to be bespoke for each research community.

Here, we explore the sex data gap in the context of human circadian physiology and sleep research. The field focuses on the temporal organization of physiology and behaviour at a daily scale, including rest-activity cycles, diurnal changes in hormone levels and cognitive performance, and the non-visual effects of light. We first describe primary findings on sex differences in circadian physiology and sleep. Next, we discuss the sex data gap in circadian and sleep research based on an analysis of over 150 papers on the non-visual effects of light, and finally we outline recommendations emerging from a virtual workshop on the biomedical implications of sex differences in sleep and circadian physiology (held in June 2020).

While we distinguish between gender identity (how individuals and groups perceive themselves e.g. men, women, non-binary,) and sex (the biological attributes that distinguish organisms as female, male or intersex) we note that these terms are often used interchangeably and wrongly in the literature [8]. Yet, in biology, sex describes differences in sexual characteristics that go beyond reproductive functions. Furthermore, we acknowledge that there is very little to no research about intersex individuals within circadian physiology and sleep, constituting an important gap. Addressing it may contribute to better granularity and understanding of sex-differentiated biological mechanisms and responses. When reporting on results from the literature, we use the terms used by the researchers in these 
studies, as we are unable to know whether participants were asked about their sex or their gender.

\section{Sex differences in sleep and circadian physiology}

Human circadian and sleep physiology features well-established sex differences: for instance, circadian timing is phase-advanced (earlier) in female compared to male individuals, as seen in the core body temperature minimum and evening rise in melatonin $[9,10]$. Female individuals also have a shorter circadian period of the temperature and melatonin rhythms [11], and larger amplitude of the melatonin rhythm [10 ]. Furthermore, sex differences exist in chronotype, the circadian continuum of early ('larks') to late ('owls') diurnal preference [21], such that more male individuals are late types than females [12-15]. With regard to sleep, female individuals have an earlier timing of sleep, longer sleep duration and more slow-wave sleep $[13,16]$.

More recently, sleep regularity - the day-to-day consistency in sleep timing and duration has emerged as an important factor in health [17]. Irregular sleep is associated with cardiovascular disease [18], inflammation [19], metabolic disorders [20-22], mental health conditions [23, 24], and cognitive impairment[25]. The data on sex differences are mixed with reports ranging from no sex differences [26-28] to more irregular sleep in female [29$31]$ or in male individuals $[32,33]$. Chronotype may account for these inconsistencies as 'owls' tend to be more irregular sleepers $[11,12]$. Indeed, in re-visiting three published datasets $[14,34,35]$, more male individuals were found to be irregular sleepers than females when both were a later chronotype.

Finally, while data remain sparse, the adverse health effects of sleep irregularity itself may also differ between the sexes. To the best of our knowledge, only one study examined this question [32], finding that variability in sleep duration was significantly associated with weight gain in male but not female students. Overall, despite the far-reaching health implications, sex differences in sleep and circadian physiology remain under-researched.

\section{Impact of sex differences in sleep and circadian physiology in a non-clinical setting.} Perhaps the most observable effect of sex differences in sleep and circadian physiology in a non-clinical setting is in shift work, a ubiquitous facet of modern society. Shift workers (approximately 50\% of which are women) account for about a third of the workforce in North 
113 America and Europe, [36]. Women have higher injury rates during night work than men,

114 despite the injury rates between men and women being similar in day workers [37]. The

115 physiological mechanisms underlying this difference remains unclear, partly due to a lack of

116 research on the female circadian system. An exception is a recent study on sex differences in

117 the effects of acute sleep deprivation on alertness [40]. This work showed that women in the

118 follicular phase of their menstrual cycle had more sleep loss-related alertness failure than

119 men, whereas there were no differences between women in the luteal phase and men [38].

120 This powerful influence of sex hormones and the menstrual cycle in female individuals

121 highlight the pressing need to consider sex differences in biomedical research.

123 Impact of sex differences in sleep and circadian physiology in a clinical setting. Evidence is

124 converging that sex differences in sleep and circadian phenotypes play a role in medical

125 conditions and should therefore be considered in medical treatments and interventions. The 126 emerging field of chronotherapeutics or chronotherapy [39-41] focuses on medical treatment 127 approaches that incorporate a patient's circadian phase, or at least time of day, into the 128 treatment regime. Here, we highlight a key therapeutic area, cancer treatment, in which sex129 specific differences in underlying circadian mechanisms affect outcomes.

131 Sex and age profoundly impact chemotherapy efficacy and tolerability. Female patients are more susceptible than their male counterparts to the side effects of widely used anticancer drugs [42-44] [45], and they can experience more frequent and severe toxicities from chemotherapy protocols due to sex differences in pharmacokinetics and pharmacodynamics [46]. Across the 24-hour cycle, the molecular circadian clock rhythmically controls drug 136 bioactivation, detoxification and transport while the circadian timing system as a whole regulates drug absorption, distribution, metabolism and excretion [47]. In experimental models, this results in strong circadian changes in the tolerability and efficacy of over 50 anticancer medications, indicating that timing is a critical factor [47, 48]. A study examining colorectal cancer - the third cause of cancer deaths worldwide - showed that the intravenous delivery of the drug 5-FU leucovorin (5-FU-LV) at a constant rate resulted in circadian

142 changes in drug concentration in plasma [49, 50]. Most importantly, female patients had 143 reduced 24-hour mean and circadian amplitude of the 5-FU body clearance compared to their 144 male counterparts [51]. Furthermore, peak delivery at $1 \mathrm{pm}$ or $4 \mathrm{pm}$ for oxaliplatin (another 145 anticancer drug) and at $1 \mathrm{am}$ or $4 \mathrm{am}$ for 5-FU-LV proved to be least toxic by up to six-fold in 
male patients, whilst optimal timing was located six hours later in female patients [52]. Thus, optimal drug timing and optimal drug doses can differ according to sex [63]. While the underlying mechanisms appear to involve sex differences in molecular clock function, their links with chrono-pharmacological determinants prompt further investigation.

\section{The sex data gap in sleep and circadian physiology}

The sex data gap exists both in in vivo [67] and in vitro research [68] and is apparent even in diseases that predominantly affect women [20]. Critically, the sex gap is not just restricted to inclusion at the experimental design stage: researchers frequently ignore sex as a factor in the analysis, even when males and females are included in the study [7].

Apart from vision, light plays a critical role in regulating physiology and behaviour via its influence on the circadian system. These effects are mediated by a multi-component photoreceptor system consisting of rods, cones and intrinsically photosensitive retinal ganglion cells in the eye that transmit information to the circadian clock via the retinohypothalamic tract.

To ascertain whether there is a sex data gap in sleep and circadian physiology research, we focused on the non-visual effects of light on human physiology and behaviour - including how it suppresses melatonin and shifts the circadian system. This topical research area has applicability in lighting standards, regulations and guidelines [53, 54], and various efforts are underway to incorporate scientific data from this research area into building recommendations. This highlights a pressing need to understand sex bias in this field.

A preliminary literature search identified 545 papers, which were evaluated against a list of exclusion criteria (see Methods for full details), yielding a total of 180 articles. In this specific analysis, we focused on the reported sex of participants, although in many instances the terms sex and gender were used interchangeably. Each paper was then reviewed by a single reviewer to determine if participant sex and numbers were reported, and where possible, the proportion of female participants was calculated. Of the papers assessed, $14(7.77 \%)$ did not give sufficient information on the sex breakdown of participants for this to be determined. In the remaining 166 articles, females comprised an average of $33.9 \%$ of the sample. Seven papers reported studying exclusively female participants, while 56 papers reported studying only males. Figure 1 shows the proportion of female study participants as a function of the publication year, calculated from the per-sex participant sample sizes. We conducted 
binomial tests to investigate the possibility of deviations from a balanced distribution of sexes, finding a large proportion of studies using only male volunteers. Interestingly, for later years, there were fewer female-only studies (but also fewer studies in total). While this may represent a shift towards more sex-inclusive recruitment, it also means that large parts of the cited literature are based on imbalanced participant samples.

Next, we examined studies that exclusively involved male or female participants ( $n=63)$. Of these, only eleven (17.5\%) provided text to justify this sample choice. For studies with only male participants the justifications included female physiology being subject to confounding factors such as menstruation $(n=3)$, research into the other sex being unnecessary due to previously published observations $(n=3)$, the study involving a sex-specific condition $(n=2)$, not being able to recruit females with a specific genetic polymorphism $(n=1)$, the study being a case study ( $\mathrm{n}=1)$, and the study being conducted in a location (field station) with only male staff $(n=1)$. We found some evidence that the number of females increased over time, with publication year and proportion of female participants being correlated $(r(164)=0.17$, $\mathrm{p}=0.02895$ ). Interestingly, the total sample size correlates with the fraction of female participants in a given study $(\mathrm{r}(164)=0.3, \mathrm{p}=0.00008$; Spearman's correlation): larger studies seem to recruit more balanced samples.

In summary, we find a sex data gap in the literature on the non-visual effects of light, which needs to be considered in current efforts to translate research findings in the 'real world'. A detailed analysis of a larger set of research articles is ongoing [55].

Misconceptions underlying sex bias. One of the important aspects of an experimental design is to simplify a complex world to generate a testing space where cause and effect can be isolated. This approach is necessary to generate 'doable problems', allowing researchers to better understand the mechanisms that underlie a biologically intricate world [56]. In animal research, this simplification has led to studying one sex and strain in one batch, an approach supported by an interpretation of the 'Reduce' element of the 3R ethical framework. Historically, this has been conceived as a requirement for minimizing the number of animals in a single experiment, thus encouraging researchers to generate a narrow testing space before extrapolating and generalizing the results. Male animals were consistently selected due to the belief that the sex hormone cycle in females would lead to greater variability in the data, which would then require a larger number of female animals to achieve the same statistical power [7]. A recent meta-analysis looking at 9932 traits found that the variability 
210 seen in female mice was not greater than for male mice - and in some cases was less - yet the 211 legacy remains [57].

212 A related misconception is that studying both males and females requires the sample size to 213 be doubled. Indeed, the analysis is not conducted independently for each sex; rather a 214 regression analysis is used to explore the variation in the outcome variable of interest after 215 accounting for effect of sex. Another benefit is that this approach also includes a statistical 216 test for whether the treatment effect depends on sex. As regression analysis does not pool the 217 data, the variance introduced by sex is accounted for, and the sensitivity for a treatment effect 218 is minimally impacted by the inclusion of two sexes. The statistical test for the main 219 treatment effect reveals the average treatment effect across the two sexes, and the interaction 220 term shows how the treatment effect differs for the two sexes. The power for the main effect will be impacted when the treatment effect goes in the opposite direction for the sexes (crossed effect) but then the power to detect an interaction will increase. Biologically, crossed effects are rare, as shown in a large study assessing the prevalence of sexual dimorphism [58]. In these situations, the treatment effect must be estimated for each sex individually. This potential situation may appear concerning to some, but it simply provides more evidence for the need to study both sexes to avoid misunderstanding biology. Notably, the ongoing misconceptions about including female individuals in research have become part of the implicit scientific practice, and they are passed on to future generations of researchers. To curtail this, we point to the National Institutes of Health $(\mathrm{NIH})$ guidelines which stipulate that male and female sexes should be included. Furthermore, rather than automatically powering to test for an interaction, we suggest that the average treatment effect represents both sexes, and a sex-disaggregated analysis would reveal possible large differences.

233 Sometimes, researchers propose studying one sex at the time, but it is important to collect 234 data on both male and female individuals simultaneously to test how the treatment interacts with sex. If data is collected independently for the two sexes, it becomes impossible to determine whether differences in estimate emerge due to sample variation or because the effect depends on sex.

A common pushback is that other sources of variation, such as age, should be considered: why should sex be the variable that is prioritized? Conducting an experiment means simplifying a complex biological world that features many sources of variation into a testing space, before generalizing the findings to reach broader conclusions. In biomedical research, the target population will be, on average, $50 \%$ male and $50 \%$ female, and it is becoming clear 
that variations between male and female physiology extend beyond hormonal differences.

244 Therefore, as a rule, sex should be the first variable to be included to significantly increase generalizability - except, as discussed in the NIH guidelines, for cases such as the study of sex-specific conditions or phenomena.

248 Understanding the research landscape and identifying opportunities for 249 change

250 In a three-part virtual workshop held in June 2020, the authors of this paper explored practices, barriers, and challenges in designing and executing inclusive research in circadian physiology and sleep research. All materials from the workshop, including the recordings and the programme, are available under the CC-BY license [59-61].

254 The workshop series comprised three 90-minute sessions held a week apart and included invited talks as well as interactive sessions. The workshop was advertised through a range of channels, including Twitter, the UK Clock Club listserv, and the personal networks of the organisers and speakers. A total of 275 participants registered for the entire workshop. Across the three workshops, between 38 and 94 attendees participated in the interactive sessions, with approximately four out of five participants being researchers (82 out of 94 in Workshop 1, 47 out of 60 in Workshop 2 and 31 out of 38 in Workshop 3).

We used the web platform Mentimeter to implement polling amongst participants as well as open-ended questions. Prior to participating in the interactive sessions, attendees were informed that their responses would be used for write-up and published as a peer-reviewed article. Attendees were free to not participate in the interactive sessions. No personal data were collected as part of the interactive Mentimeter sessions. We combined yes/no, ranking and open-ended questions throughout the interactive sessions to vary the response modality. The results discussed below were selected from the results, which can be viewed in full on the Open Science Framework page. The number of responses to individual questions varied somewhat due to dropout during the interactive session as well as a time-limited response window; the total number of responses in the participatory parts are given on the bottom right-hand corner of the Materials document. 
In the first workshop, we explored sex as a variable in research. In an interactive polling segment following this workshop, only $58 \%$ of respondents (out of 100) indicated previously analyzing data in a sex-disaggregated fashion. However, 88.1\% (out of 101) agreed that sex could be a variable in their research, showing the large scope for sex-disaggregated analyses. Of note, sex was identified as just one of many characteristics contributing to individual differences in research results, alongside age chronotype, mental health status, genetics, body mass index and prior light exposure. When asked for the most pressing research questions involving individual differences, the answers ranged from developmental and lifespan factors to more fundamental research questions with no obvious individual-difference angle. The video recording for Workshop 1 is available here, and the materials related to the participatory part are available here.

\section{Workshop 2: Understanding impact.}

The second workshop focused on understanding the real-world impact of the participants ' research. In the interactive polling segment following this workshop, participants indicated that their research could mostly influence precision and personalized medicine, occupational timing and shift-rota planning, and guidelines for indoor a 'circadian' lighting.

When asked to identify the biggest barriers to addressing sex bias in research, research money or funding and time were the most mentioned factors, followed by guidelines and policies. This indicates a scope for funding agencies to specifically address researchers 'need for funding, as well as an opportunity for institutions, funders, professional bodies, learned societies and journals to develop clear guidance (see Box 1 for an example of a journal implementing a specific policy; and Figure 2). The video recording for Workshop 2 is available here, and the materials related to the participatory part are here.

\section{Workshop 3: Understanding change.}

The third workshop explored factors that would facilitate change in research. In the interactive polling segment, when asked to rank sources for guidance on sex-difference analysis, the participants first mentioned research institutes and universities, then societies and professional bodies and finally funders and publishers.

302 In further exploring the role of funders, the top three priorities for participants were: (1)

303 provision of training and guidance to incorporate sex and gender analysis; (2) allocation of 304 funding within regular grant mechanisms ring-fenced for sex and gender analysis; and (3) 
simply more allocation of funds in regular research grants. Additionally, collaboratively developed guides, research toolkits, training programmes from societies and professional bodies were also indicated as facilitators of change.

When asked what researchers could personally do, three actionable items emerged: (1) inclusion of sex and gender analysis as a central step in research; (2) learning from peers and with examples; and (3) upskilling in the requisite statistical techniques. The video recording for Workshop 3 is available here, and the materials related to the participatory part are available here.

\section{Recommendations}

Guiding principles to close the sex data gap. Based on the workshop content and discussions, we propose the following guiding principles to address the sex data gap in biomedical research, and to build an evidence base which is better inclusive of sex and gender. The central tenet includes sex and gender analysis as an essential component of research design. The specific recommendations are:

1. Design research to be inclusive and accessible. In many cases, research is designed exclusively by researchers who may not necessarily have sufficient expertise on how to make their study inclusive and accessible. An important step is reaching clarity in recording and reporting participant sex and gender. As an example, one research team reporting the sex of participants may use participant-derived responses on a questionnaire or intake form, and another group may use the sex assigned at birth, based, for instance, on an ID card. While these could give congruent answers, they represent different types of information. Wider engagement with definitions of sex and gender and questions surrounding this topic within a research group or researcher community could lay the groundwork for making research more inclusive and accessible. As a formalised way to ensure inclusivity, we also suggest that research participants be integrated in the research planning process through Patient and Public Involvement (see Box 2) or similar mechanisms.

2. Implement recruitment strategies that lead to a sex-balanced sample. This includes wide advertisement of research studies, and tailoring recruitment strategies by engaging with patients, participants and the general public, for example through Patient and Public Involvement mechanisms (see Box 2). Given fixed resources, recruiting a sex-balanced sample does not simply mean doubling the sampling size, but merely recruiting a sample 
with $50 \%$ female and $50 \%$ male participants. A balanced design is recommended to ensure the resulting statistical analysis is robust and that the variance can be decomposed to the factors of interest without confounding these [62]. While exceptions to this principle may arise from sex-specific research questions, as a general guiding principle there is little to argue against. Furthermore, this will allow sex to be included as a factor in the analysis without compromising sensitivity to a generalizable main effect.

3. Use data visualization to grasp the effect of sex. An informal visualization in the early stages of analyses can be used to ascertain sex-difference trends, which can then be followed up with more rigorous statistical testing.

4. Implement statistical analyses that include sex as a factor and/or perform group analyses by sex, where possible. Sex can be included as a factor or a covariate in analyses, or an alternative strategy can be to perform a group analysis by sex. Both require a good understanding of effect sizes and statistical power. Researchers should seek to upskill in statistics to develop advanced analytic strategies.

5. Make participant-level data open and available to facilitate future meta-analytic efforts. This step requires data to be available, which many journals now mandate. The large, combined sample size afforded by the wide availability of data can enable a sexrelated effect to be more readily detectable. We also suggest that researchers should include tables reporting the primary data and participant meta-data as supplementary information in articles. A recent analysis of open science practices in circadian rhythms and sleep research journals [63] has indicated an opportunity to mandate data sharing in journal policies. Journal policies requiring participant-level data sharing could facilitate future analyses incorporating sex.

While none of these actions will suffice on their own, each will contribute to closing the sex data gap. Of course, the research ecosystem not only includes individual researchers but also institutions of varying sizes. We present multiple actions that can be adopted by institutions, funders, as well as professional bodies, learned societies, journals in Figure 2. These actions were developed from an interactive segment of Workshop 3. 
Box 1: Example journal policy to addressing sex bias

367 Amrita Ahluwalia, Editor-in-Chief of British Journal of Pharmacology (BJP)

368 In 2018, the British Journal of Pharmacology identified the issue of sex bias in pharmacological research as a critical area for attention with respect to the work published in

370 the journal. This came following an internal survey of our published work coupled with

371 recognition of the activities and actions of the National Institutes of Health, in the US, raising

372 the profile of this important issue [64]. We discovered that in addition to a prevailing

373 reluctance to use female individuals in experimental research, both in vivo and in vitro, there

374 was the unsurprising omission of detail regarding the sex of the source for experimental work

375 involving primary cell culture [6].

376 To address these issues, we introduced a number of initiatives, including: (1) publishing a 377 themed issue in BJP containing a number of reviews and original articles focused on sex 378 differences in pharmacology; (2) bringing together a collection of articles from all of the 379 journals owned by the BPS in a virtual issue focused on sex; and, most importantly, (3) the 380 elaboration and publication of guidelines for original research published in BJP. The aim of 381 this guidance is to ensure that sex as an experimental variable is no longer ignored in articles 382 published in BJP, but also to provide researchers with the tools to adapt their experimental 383 design to accommodate for sex.

384 A key aspiration, of course, is that both male and female subjects are used as a default design 385 in the experimental work detailed in all manuscripts submitted to the journal, but we do not mandate this at present. Our hope is that by insisting on consideration of these issues within any submitted work, we raise the profile of the issue, and that this organically leads to change. Of course, it is the responsibility of those who work with the journal to ensure that change does indeed occur. Indeed, there are many examples where such an advisory approach with other important issues related to transparency and reproducibility appear to have failed $[65,66]$. Yet our experience in such approaches at BJP - for instance, with our guidelines on design and analysis [67] - gives us strong hope that change will take place. We plan to conduct surveys of published material annually to assess this, and we will publish the outcome of these audits. 


\section{inclusive}

397 Patient and Public Involvement (PPI) [68-70] is defined as research carried out 'with' or 'by'

398 patients, those who have experience of a condition, and the broader public in general. PPI is a

399 term that is largely used in the UK research landscape, but similar initiatives may exist in

400 different countries. PPI differs markedly from engagement and participation; this refers to

401 various types of interactions with people with a condition (such as providing information and

402 knowledge in research) as well as surveying what people understand about a particular

403 condition regardless of whether they experience it, or exploring what should be prioritized in

404 basic or clinical research on that condition. Involvement, on the other hand, implies a more

405 active collaboration between researchers, and the target group - and in some cases the

406 general public - that helps shape the design of a research project. At different levels, all these

407 interactions provide opportunities for dialogue and bring research to those directly impacted

408 by conditions, and the public. This, in turn, helps increase diversity - including, but not

409 limited to, making research more inclusive with respect to sex and gender.

410 Engaging with the general public and with patients is now often asked by charities and 411 research funding organizations but should be considered beyond being a box-ticking exercise.

412 PPI will very likely impact the design of research projects by identifying what is vital to 413 patients and society, and why. In turn, this will help to identify gaps in our understanding of 414 the disease or condition in question thereby increasing research quality. This can help 415 prioritize research areas and lead to research that is better aligned with the patient's and 416 public's interests. For example, the James Lind Alliance Priority Setting Partnerships is a 417 non-profit initiative bringing patients, carers and clinicians together to identify and prioritise 418 unresolved questions or evidence uncertainties they consider important. In this way, research 419 funders become aware of what matters most to the people who use their research in their 420 everyday lives. PPI will also help the target group to better understand research, and give an 421 often unique opportunity for researchers - especially discovery scientists - to understand 422 patients' reality and perspective. 
426 To implement a breadth-first search for identifying relevant papers, we employed a pragmatic hybrid strategy, identifying relevant articles through three main sources, as listed in Table 1.

428 We conducted a citation search of three key, recent reviews [71-73] on the acute effects of light, producing a total of 88 papers of which 83 were included in the present analysis. We carried out a search for papers specifically discussing the melatonin-suppressive effects of light in SCOPUS (search carried out on 22 October 2019) through the search term "TITLEABS-KEY ( ( light AND melatonin AND suppress*) ) AND ( LIMIT-TO ( DOCTYPE , "ar" ) ) AND ( LIMIT-TO ( LANGUAGE , "English" ) )" (search carried out on 22 October 2019). Limiting the analysis to papers with a minimum of 30 citations, we identified 359 further papers (94 of which were included). Finally, relevant systematic reviews were identified in the Cochrane Library through the search terms "(light AND (circadian OR sleep OR alertness)", generating 24 results with 6 relevant for the present analysis. A citation search was again conducted, generating a further 98 papers (of which 3 were included). Overall, a total of 545 papers were identified and analyzed, as shown in Table 1.

Inclusion and exclusion criteria. Papers were excluded where the following exclusion criteria applied, leaving a total of 180 papers for the present analysis:

1. Studies that do not assess the acute effect of light: including those looking at longitudinal exposures or habits rather than controlled light exposure within a specified time frame, e.g. cohort and case-control studies were excluded;

2. Studies in which the primary outcome measure did not relate to circadian physiology (e.g. the role of light exposure in treating affective disorders);

3. Studies assessing the effects of interventions other than light exposure, e.g. sleep deprivation or magnetic field exposure. In papers involving multiple studies, only those assessing the acute effects of light were included, with other studies excluded;

4. Studies for which the PDF of the paper could not be obtained, or could not be obtained in English;

5. Studies primarily focusing on non-human animals;

6. Review papers, opinion pieces or commentaries not including any primary data; 
7. Studies not based on measurements taken from human participants, e.g. in vitro studies or mathematical models. Measurements of human materials such as blood or retinal cells were considered to be from human participants if the intervention (light exposure) was carried out before the material was isolated from participants, but they were excluded if measurements were taken after the materials were obtained;

8. Research involving participants under the age of 18 ;

9. Studies in which variables were not manipulated (i.e., naturalistic or observational studies);

10. Field studies, in which variables were manipulated outside of a laboratory setting.

\section{Papers were not excluded based on participant disease status or outcome measure. No upper} limit was set for participant age. In coding the articles, we did not make a distinction between sex and gender, as these are conflated in the literature.

\begin{tabular}{|c|c|c|c|c|}
\hline Database & Search strategy & Source paper & $\begin{array}{l}\text { Articles } \\
\text { considered }\end{array}$ & $\begin{array}{l}\text { Articles } \\
\text { included }\end{array}$ \\
\hline
\end{tabular}

\begin{tabular}{|c|c|c|c|c|c|}
\hline- & \multicolumn{2}{|l|}{-} & Brown (2020) [71] & 19 & 18 \\
\hline- & \multicolumn{2}{|l|}{-} & Lok et al. (2018) [73] & 20 & 20 \\
\hline- & \multicolumn{2}{|l|}{-} & $\begin{array}{l}\text { Souman et al. (2018) } \\
{[72]}\end{array}$ & 49 & 45 \\
\hline SCOPUS & \multicolumn{2}{|c|}{ Citation count } & - & 359 & 94 \\
\hline \multirow[t]{2}{*}{ Cochrane } & \multirow{2}{*}{\multicolumn{2}{|c|}{$\begin{array}{lr}\text { (light } & \text { AND } \\
\text { (circadian } & \text { OR } \\
\text { sleep } & \text { OR } \\
\text { alertness)" } & \end{array}$}} & $\begin{array}{l}\text { Pachito et al (2018) } \\
{[74]}\end{array}$ & 5 & 0 \\
\hline & & & $\begin{array}{l}\text { Forbes et al. (2014) } \\
{[75]}\end{array}$ & 13 & 0 \\
\hline
\end{tabular}




Montgomery \& $0 \quad 0$

Dennis (2002) [76]

Tuunainen, Kripke \& 49 3

Endo (2014) [77]

Slanger et al. (2016) 21 0 [78]

Dennis \& Donswell 10 0 (2013) [79]

467

468 Table 1: Articles included in the meta-analysis. 
1. Criado-Perez, C., Invisible women: data bias in a world designed for men. 2019, New York: Abrams Press. xv, 411 pages.

2. Buitendijk, S. and K. Maes, Gendered research and innovation: integrating sex and gender analysis into the research process [Advice paper]. League of European Research Universities, 2015.

3. Lee, S.K.J.B.r., Sex as an important biological variable in biomedical research. 2018. 51(4): p. 167.

4. Clayton, J.A. and F.S. Collins, NIH to balance sex in cell and animal studies. Nature, 2014. 509(7500): p. 282-3.

5. Rippon, G., et al., Journal of neuroscience research policy on addressing sex as a biological variable: comments, clarifications, and elaborations. 2017. 95(7): p. 13571359.

6. Docherty, J.R., et al., Sex: A change in our guidelines to authors to ensure that this is no longer an ignored experimental variable. British journal of pharmacology, 2019. 176(21): p. 4081.

7. Karp, N.A. and N. Reavey, Sex bias in preclinical research and an exploration of how to change the status quo. British journal of pharmacology, 2018.

8. Tannenbaum, C., et al., Sex and gender analysis improves science and engineering. Nature, 2019. 575(7781): p. 137-146.

9. Boivin, D.B., et al., Diurnal and circadian variation of sleep and alertness in men vs. naturally cycling women. Proc Natl Acad Sci U S A, 2016. 113(39): p. 10980-5.

10. Cain, S.W., et al., Sex differences in phase angle of entrainment and melatonin amplitude in humans. J Biol Rhythms, 2010. 25(4): p. 288-96.

11. Duffy, J.F., et al., Sex difference in the near-24-hour intrinsic period of the human circadian timing system. Proc Natl Acad Sci U S A, 2011. 108 Suppl 3: p. 15602-8.

12. Fischer, D., et al., Chronotypes in the US - Influence of age and sex. PLoS One, 2017. 12(6): p. e0178782.

13. Roenneberg, T., et al., A marker for the end of adolescence. Curr Biol, 2004. 14(24):

14. Fischer, D., C. Vetter, and T. Roenneberg, A novel method to visualise and quantify circadian misalignment. Sci Rep, 2016. 6: p. 38601.

15. Phillips, A.J.K., et al., Irregular sleep/wake patterns are associated with poorer academic performance and delayed circadian and sleep/wake timing. Sci Rep, 2017. 7(1): p. 3216.

16. Dijk, D.J., D.G. Beersma, and G.M. Bloem, Sex differences in the sleep EEG of young adults: visual scoring and spectral analysis. Sleep, 1989. 12(6): p. 500-7.

17. Bei, B., et al., Beyond the mean: A systematic review on the correlates of daily intraindividual variability of sleep/wake patterns. Sleep Med Rev, 2016. 28: p. 10824.

18. Yoon, D.Y., et al., Sex bias exists in basic science and translational surgical research. Surgery, 2014. 156(3): p. 508-516.

19. Okun, M.L., et al., Sleep variability, health-related practices, and inflammatory markers in a community dwelling sample of older adults. Psychosom Med, 2011. 73(2): p. 142-50. 
20. Patel, S.R., et al., The association between sleep patterns and obesity in older adults. Int J Obes (Lond), 2014. 38(9): p. 1159-64.

21. Chontong, S., S. Saetung, and S. Reutrakul, Higher sleep variability is associated with poorer glycaemic control in patients with type 1 diabetes. J Sleep Res, 2016. 25(4): p. 438-44.

22. Spruyt, K., D.L. Molfese, and D. Gozal, Sleep duration, sleep regularity, body weight, and metabolic homeostasis in school-aged children. Pediatrics, 2011. 127(2): p. e34552.

23. Lemola, S., T. Ledermann, and E.M. Friedman, Variability of sleep duration is related to subjective sleep quality and subjective well-being: an actigraphy study. PLoS One, 2013. 8(8): p. e71292.

24. Vanderlind, W.M., et al., Sleep and sadness: exploring the relation among sleep, cognitive control, and depressive symptoms in young adults. Sleep Med, 2014. 15(1): p. 144-9.

25. McBean, A.L. and H.E. Montgomery-Downs, Timing and variability of postpartum sleep in relation to daytime performance. Physiol Behav, 2013. 122: p. 134-9.

26. Kaufmann, C.N., et al., Clinical significance of mobile health assessed sleep duration and variability in bipolar disorder. J Psychiatr Res, 2016. 81: p. 152-9.

27. $\mathrm{Xu}, \mathrm{X}$., et al., Habitual sleep duration and sleep duration variation are independently associated with body mass index. Int J Obes (Lond), 2018. 42(4): p. 794-800.

28. Minors, D., et al., The effects of age upon some aspects of lifestyle and implications for studies on circadian rhythmicity. Age Ageing, 1998. 27(1): p. 67-72.

29. Mezick, E.J., et al., Intra-individual variability in sleep duration and fragmentation: associations with stress. Psychoneuroendocrinology, 2009. 34(9): p. 1346-54.

30. Dillon, H.R., et al., Variability in self-reported normal sleep across the adult age span. J Gerontol B Psychol Sci Soc Sci, 2015. 70(1): p. 46-56.

31. Lunsford-Avery, J.R., et al., Validation of the Sleep Regularity Index in Older Adults and Associations with Cardiometabolic Risk. Sci Rep, 2018. 8(1): p. 14158.

32. Roane, B.M., et al., What Role Does Sleep Play in Weight Gain in the First Semester of University? Behav Sleep Med, 2015. 13(6): p. 491-505.

33. Yetish, G., H. Kaplan, and M. Gurven, Sleep variability and nighttime activity among Tsimane forager-horticulturalists. Am J Phys Anthropol, 2018. 166(3): p. 590-600.

34. Fischer, D., et al., Irregular sleep and event schedules are associated with poorer self-reported well-being in US college students. Sleep, 2020. 43(6).

35. Keller, L.K., et al., Not later, but longer: sleep, chronotype and light exposure in adolescents with remitted depression compared to healthy controls. Eur Child Adolesc Psychiatry, 2017. 26(10): p. 1233-1244.

36. Kervezee, L., A. Shechter, and D.B. Boivin, Impact of Shift Work on the Circadian Timing System and Health in Women. Sleep Med Clin, 2018. 13(3): p. 295-306.

37. Safe Work Australia, Australian work-related injury experience by sex and age, 2009-10. 2012, Canberra: Creative Commons.

38. Vidafar, P., et al., Increased vulnerability to attentional failure during acute sleep deprivation in women depends on menstrual phase. Sleep, 2018. 41(8).

39. Shuboni-Mulligan, D.D., et al., Radiation chronotherapy-clinical impact of treatment time-of-day: a systematic review. J Neurooncol, 2019. 145(3): p. 415-427.

40. Smolensky, M.H., et al., Bedtime Chronotherapy with Conventional Hypertension Medications to Target Increased Asleep Blood Pressure Results in Markedly Better Chronoprevention of Cardiovascular and Other Risks than Customary On-awakening Therapy. Heart Fail Clin, 2017. 13(4): p. 775-792. 
600

601

602

603

604

605

606

607

608

609

610

611

612

613

614

41. Dijk, D.J. and J.F. Duffy, Novel Approaches for Assessing Circadian Rhythmicity in Humans: A Review. J Biol Rhythms, 2020: p. 748730420940483.

42. Milano, G., et al., Influence of sex and age on fluorouracil clearance. J Clin Oncol, 1992. 10(7): p. 1171-5.

43. Stein, B.N., et al., Age and sex are independent predictors of 5-fluorouracil toxicity. Analysis of a large scale phase III trial. Cancer, 1995. 75(1): p. 11-7.

44. Chansky, K., J. Benedetti, and J.S. Macdonald, Differences in toxicity between men and women treated with 5-fluorouracil therapy for colorectal carcinoma. Cancer, 2005. 103(6): p. 1165-71.

45. Cristina, V., et al., Association of Patient Sex With Chemotherapy-Related Toxic Effects: A Retrospective Analysis of the PETACC-3 Trial Conducted by the EORTC Gastrointestinal Group. JAMA Oncol, 2018. 4(7): p. 1003-1006.

46. Gandhi, M., et al., Sex differences in pharmacokinetics and pharmacodynamics. Annu Rev Pharmacol Toxicol, 2004. 44: p. 499-523.

47. Dallmann, R., A. Okyar, and F. Levi, Dosing-Time Makes the Poison: Circadian Regulation and Pharmacotherapy. Trends Mol Med, 2016. 22(5): p. 430-445.

48. Levi, F., et al., Circadian timing in cancer treatments. Annu Rev Pharmacol Toxicol, 2010. 50: p. 377-421.

49. Petit, E., et al., Circadian rhythm-varying plasma concentration of 5-fluorouracil during a five-day continuous venous infusion at a constant rate in cancer patients. Cancer Res, 1988. 48(6): p. 1676-9.

50. Fustin, J.M., et al., Rhythmic nucleotide synthesis in the liver: temporal segregation of metabolites. Cell Rep, 2012. 1(4): p. 341-9.

51. Bressolle, F., et al., Circadian rhythm of 5-fluorouracil population pharmacokinetics in patients with metastatic colorectal cancer. Cancer Chemother Pharmacol, 1999. 44(4): p. 295-302.

52. Levi, F., et al., Implications of circadian clocks for the rhythmic delivery of cancer therapeutics. Adv Drug Deliv Rev, 2007. 59(9-10): p. 1015-35.

53. CIE, CIE Position Statement on Non-Visual Effects of Light-Recommending Proper Light at the Proper Time (2nd edition, October 2019). 2019.

54. WELL, WELL v2. 2020.

55. Tir, S. and M. Spitschan, Study population characteristics in sleep research and chronobiology: Protocol for a systematic review. 2020.

56. Gompers, A., Dec 10 Dec 10 Three Years In: "Sex as a Biological Variable" Policy in Practice-and an Invitation to Collaborate.

57. Prendergast, B.J., K.G. Onishi, and I. Zucker, Female mice liberated for inclusion in neuroscience and biomedical research. Neuroscience \& Biobehavioral Reviews, 2014. 40: p. 1-5.

58. Karp, N.A., et al., Prevalence of sexual dimorphism in mammalian phenotypic traits. Nat Commun, 2017. 8: p. 15475.

59. Spitschan, M., et al., Sex differences in circadian rhythms and sleep - Understanding difference (15 June 2020). 2020.

60. Spitschan, M., et al., Sex differences in circadian rhythms and sleep - Understanding change (29 June 2020). 2020.

61. Spitschan, M., et al., Sex differences in circadian rhythms and sleep - Understanding impact (22 June 2020). 2020.

62. Collins, L.M., J.J. Dziak, and R. Li, Design of experiments with multiple independent variables: a resource management perspective on complete and reduced factorial designs. Psychol Methods, 2009. 14(3): p. 202-24. 
615

616

617

618

619

620

621

622

623

624

625

626

627

628

629

630

631

632

633

634

635

636

637

638

639

640

641

642

643

644

645

646

647

648

649

650

651

652

653

654

655

656

657

658

659

660

661

662

663
63. Spitschan, M., M.H. Schmidt, and C. Blume, Transparency and open science principles in reporting guidelines in sleep research and chronobiology journals. Wellcome Open Research, 2020. 5.

64. National Institutes of Health. NIH Policy on Sex as a Biological Variable [Archived on Internet Archive Wayback Machine, 28 July 2020]. 2020; Available from: http://web.archive.org/web/20200728094053/https://orwh.od.nih.gov/sex-gender/nihpolicy-sex-biological-variable.

65. Leung, V., et al., ARRIVE has not ARRIVEd: Support for the ARRIVE (Animal Research: Reporting of in vivo Experiments) guidelines does not improve the reporting quality of papers in animal welfare, analgesia or anesthesia. PLOS ONE, 2018. 13(5): p. e0197882.

66. Avey, M.A.-O., et al., The Devil Is in the Details: Incomplete Reporting in Preclinical Animal Research. PLOS ONE, 2016. 11(1932-6203 (Electronic)): p. e0166733.

67. Curtis, M.J., et al., Experimental design and analysis and their reporting II: updated and simplified guidance for authors and peer reviewers. British Journal of Pharmacology, 2018. 175(7): p. 987-993.

68. National Institute for Health and Care Excellence. Patient and public involvement policy. 2020; Available from: https://www.nice.org.uk/about/nice-communities/niceand-the-public/public-involvement/public-involvement-programme/patient-publicinvolvement-policy.

69. Arthritis Research UK, Patient \& Public Involvement: A researcher's guide. 2017, Chesterfield: Arthritis Research UK.

70. Bagley, H.J., et al., A patient and public involvement (PPI) toolkit for meaningful and flexible involvement in clinical trials - a work in progress. Res Involv Engagem, 2016. 2: p. 15.

71. Brown, T.M., Melanopic illuminance defines the magnitude of human circadian light responses under a wide range of conditions. J Pineal Res, 2020: p. e12655.

72. Souman, J.L., et al., Acute alerting effects of light: A systematic literature review. Behav Brain Res, 2018. 337: p. 228-239.

73. Lok, R., et al., Light, Alertness, and Alerting Effects of White Light: A Literature Overview. J Biol Rhythms, 2018. 33(6): p. 589-601.

74. Pachito, D.V., et al., Workplace lighting for improving alertness and mood in daytime workers. Cochrane Database Syst Rev, 2018. 3: p. CD012243.

75. Forbes, D., et al., Light therapy for improving cognition, activities of daily living, sleep, challenging behaviour, and psychiatric disturbances in dementia. Cochrane Database Syst Rev, 2014(2): p. CD003946.

76. Montgomery, P. and J. Dennis, Bright light therapy for sleep problems in adults aged 60+. Cochrane Database Syst Rev, 2002(2): p. CD003403.

77. Tuunainen, A., D.F. Kripke, and T. Endo, Light therapy for non-seasonal depression. Cochrane Database Syst Rev, 2004(2): p. CD004050.

78. Slanger, T.E., et al., Person-directed, non-pharmacological interventions for sleepiness at work and sleep disturbances caused by shift work. Cochrane Database Syst Rev, 2016(8): p. CD010641.

79. Dennis, C.L. and T. Dowswell, Interventions (other than pharmacological, psychosocial or psychological) for treating antenatal depression. Cochrane Database Syst Rev, 2013(7): p. CD006795. 
Figure 1. A review of the literature on the non-visual effects of light reveals a sex bias.

669

670 We analyzed a sample of the existing literature on the non-visual effects of light as a starting point for understanding the sex bias in the field. The sample included a total of 180 articles, and the breakdown of participant sex was then obtained in 166 articles. Binomial tests were conducted to evaluate the possibility that deviations from an even 50:50 sex distribution were attributable to chance alone. We implemented the Benjamini-Hochberg correction for multiple comparisons to control false-discovery rate (FDR). The proportion of female volunteers in each paper (represented by a dot) was plotted against the year of publication. Samples for which the proportion of female patients deviated significantly from 0.5 ( $\mathrm{p} \leq$

678 0.05) were determined to be biased and colour-coded as orange. The marginal histograms 679 show the numbers of papers irrespective of publication year (histogram on the right $y$ axis), 680 or irrespective of proportion (histogram on top $x$ axis). Methods for paper selection are 681 included in Methods.

683 Figure 1-Source Data File. Excel spreadsheet containing the data underlying Figure 1.

684 Figure 1-Source Code File. R code to produce Figure 1. 


Researchers and
institutes and
clinician-scientists
universities

688

689 Figure 2. Suggested actions to close the sex data gap in sleep and circadian research for 690 actors across the ecosystem. These actions were derived from an interactive session with 691 attendees $(n=38)$ during Workshop 3. 